\title{
Micro Scribing of Copper and Aluminum Thin Films in Air and Water Using Pulsed $\mathrm{Nd}^{3+}$ :YAG Laser
}

\author{
Srinagalakshmi Nammi ${ }^{1}$, Ankit K Jain ${ }^{1}$, Nilesh J Vasa ${ }^{1}$, G Balaganesan ${ }^{2}$, Anil C Mathur ${ }^{3}$ \\ ${ }^{1}$ Department of Engineering Design, Indian Institute of Technology Madras, Chennai 600036, India \\ Email:snl.nammi@gmail.com \\ 2 Department of Mechanical Engineering, Indian Institute of Madras, Chennai 600036, India \\ ${ }^{3}$ Scientist, Antenna Mechanical Design Division, Indian Space Research Organization, Ahmedabad 380015, India
}

\begin{abstract}
Micro scribing of copper and aluminum thin films in air and underwater using Q-switched $\mathrm{Nd}^{3+}:$ YAG laser has been described. Influence of de-ionized (DI) water level on the scribed micro channel depth and width has been studied for different wavelengths. Scribing was done by maintaining different levels of DI water at $1 \mathrm{~mm}, 2 \mathrm{~mm}, 3 \mathrm{~mm}, 5 \mathrm{~mm}$ and $10 \mathrm{~mm}$. At $1 \mathrm{~mm}$ DI water level and laser pulse energy of $3 \mathrm{~mJ}$, maximum ablation depth of $105 \mu \mathrm{m}$ was achieved with both $355 \mathrm{~nm}$ and $532 \mathrm{~nm}$ wavelength for aluminum. Further, the depth achieved in DI water was 6 times greater than that in air for aluminum. On the other hand, for copper film, the depth attained with the laser ablation in DI water was 7 um as compared to $4 \mathrm{um}$ in air for $1064 \mathrm{~nm}$ wavelength and $40 \mathrm{~mJ}$ of energy. In case of $355 \mathrm{~nm}$ and $532 \mathrm{~nm}$ the depth attained in air was higher than in DI water for copper. As the DI water level was increased, the depth reduced for both aluminum and copper. A comparative study of the results obtained in both underwater and air media revealed that aluminum can be machined more potently in DI water than in air. Further, theoretical modeling of the laser-material interaction in air as ambience has been discussed to estimate the recession rate by incorporating the laser ablation temperature measured using the laser induced breakdown spectroscopy technique.
\end{abstract}

DOI: $10.2961 /$ jlmn.2016.01.0009

Keywords: laser scribing, under water scribing, thin films, parabolic reflector, laser micro machining

\section{Introduction}

In communication satellites, aluminum/copper films coated with polyimide substrate are used in parabolic RF antennas. The reflector surface has evenly spaced conductive grids with size ranging in the order of microns. The size and shape of the grids influence the effective functioning of the reflector. Conventionally, the conductive grids on the polyimide substrate are fabricated by mechanical methods that involve cutting of thin metal sheets into finer strips and pasting them in parallel lines on the surface of the reflector. Research is widely carried out in search of precise and reliable fabrication processes for producing these conductive grids.

Pulsed laser ablation (PLA) has been widely studied in the past as an effective method for the ablation of thin metal films [1]. Removal of material occurs due to the interaction of the intense electromagnetic waves in the laser radiation with the work piece, leading to heating, melting and vaporization of a selective micro volume. In contrast with a conventional machine tool process, laser machining process does not involve tool wear, vibrations and burr formation; material removal depends on the optical and thermal properties of the workpiece [2]. Nano second laser pulse absorbed by the workpiece vaporizes a focused volume of material through heat conduction. The ejected vapour interacts with the incoming laser beam and forms a plasma plume [3]. Depending on the ambient conditions, the plasma plume exerts a recoil pressure, expelling the melt pool that gets redeposited at the edge of the crater or channel [4]. In order to avoid this redeposition of expelled material on the workpiece surface, many techniques are being used that includes high pressure gas/liquid. Laser coupled with high pressure water through total internal reflection shows better surface quality and reduced heat affected zone when scribing thin brass and stainless steel work pieces [5]. Underwater laser ablation of aluminum is shown as a cost effective drilling process compared to dry ablation, with improvement in the crater shape quality [6]. Metal plates of varying thicknesses have been drilled using $\mathrm{Nd}^{3+}$ :YAG laser in water and air and the material removal in under water drilling is 10 times more than in air due to the plasma confinement by water [7]. Bubble formation and collapse due to vapourization of water is shown to be an effective method for removal of debris in wet ablation process [8].

Many research groups have reported on under water laser ablation process including only infrared wavelength. However, there is a need to understand the behavior of laser ablation of $\mathrm{Al} / \mathrm{Cu}$ films for a wide range of laser wavelengths. Laser scribing of reflective materials, such as $\mathrm{Cu}$ and $\mathrm{Al}$ films has not been studied in detail. Micro machining on thin copper films was performed for three laser wavelengths $(355 \mathrm{~nm}, 532 \mathrm{~nm}$ and $1064 \mathrm{~nm})$ and the effect of intensity and processing rate on the surface morphology has been investigated in air [9,10]. Free-electron thermal conductivity is high in these metals and it makes the controlled machining of reflective materials using laser a challenging task. Aluminum has very high reflectivity (approximate $90 \%$ ) due to multi-valency of the metal that is responsible for high plasma frequency of $\mathrm{Al}$ [11]. However, there are very few reports on the same using the ns-pulsed $\mathrm{Nd}^{3+}$ :YAG laser in particular.

This paper aims at presenting certain results related to under water laser scribing using UV (355 nm), visible (532 $\mathrm{nm})$ and IR (1064 nm) wavelengths of a Q-switched, pulsed $\mathrm{Nd}^{3+}$ :YAG. The influence of various laser parameters such as laser beam profile, wavelength and ambient condition has been studied to develop laser scribing tech- 
niques for manufacturing micro-channels on $\mathrm{Al} / \mathrm{Cu}$ film deposited on a flexible substrate. Further, the laser induced breakdown spectroscopy (LIBS) technique has been proposed and demonstrated for estimating the plume temperature during the laser scribing of thin films. Theoretical modeling has also been attempted to understand the effect of different wavelengths $(355 \mathrm{~nm}, 532 \mathrm{~nm}$ and $1064 \mathrm{~nm})$ on the recession rate and ablation temperature for thin copper sample and the results are discussed.

\section{Experiment Studies}

\subsection{Experimental Setup}

A Q-switched $\mathrm{Nd}^{3+}$ :YAG laser with a pulse width of $6 \mathrm{~ns}$ and a repetition rate of $5 \mathrm{~Hz}$ was used in this study. The laser beam was directed on to the workpiece using beam alignment optics such as dichroic mirrors and a quartz lens with a focal length of $250 \mathrm{~mm}$ as shown in figure 1. Laser scribing was performed on $150 \mu \mathrm{m}$ thickness Al film and $35 \mu \mathrm{m}$ thickness $\mathrm{Cu}$ films on a $50 \mu \mathrm{m}$ thick polyimide substrate. Experiments in air medium with the Gaussian laser beam profile were performed by mounting the workpiece on a motorized $\mathrm{X}-\mathrm{Y}$ stage. On the other hand, experiments in under water medium with de-ionized water were performed by fixing the workpiece in a water beaker placed over the X-Y stage. During LIBS study, the optical emission was collected at an angle of $30^{\circ}$ by a lens of focal length $150 \mathrm{~mm}$ and was focused onto an optical fiber (core diameter $600 \mu \mathrm{m}$, NA 0.39). A spectrometer (UV-VIS2000, Ocean Optics) coupled with the fiber was used for the detection of spectral information. Surface characterization after the scribing process was carried out using a noncontact optical profilometer (Contour GT-I, Bruker) and a scanning electron microscope (Quanta 200, FEG).

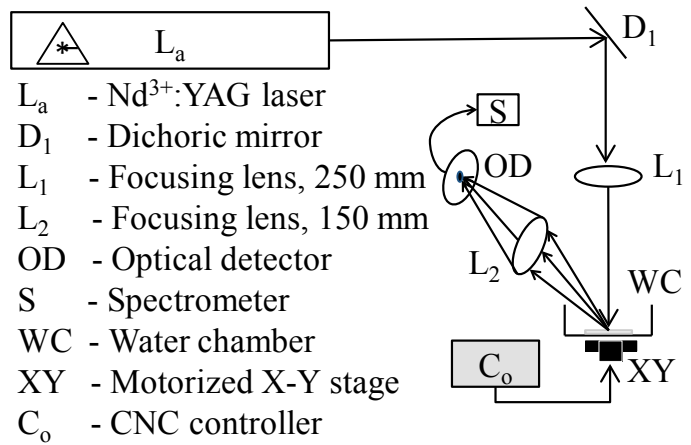

Fig. 1 Schematic of experimental setup used for laser scribing in air and under water

Table 1: Parameters and specifications used in the experiment to study the influence of wavelength and ambience

\begin{tabular}{ll}
\hline Parameters & Specifications \\
\hline Laser used & $\mathrm{Nd}^{3+}:$ YAG $(\lambda=355,532$ and \\
$1064 \mathrm{~nm})$ \\
$\begin{array}{l}\text { Pulse duration (Fre- } \\
\text { quency) }\end{array}$ & $5 \mathrm{~ns}(5 \mathrm{~Hz})$ \\
$\mathrm{Cu}$ and Al sample size & $20 \mathrm{~mm} \times 20 \mathrm{~mm}$ \\
Focal length & $250 \mathrm{~mm}$ \\
Spot size (estimated) & $\approx 200 \mu \mathrm{m}$ \\
Beam overlap & $95 \%$ \\
Ambient condition & $\begin{array}{l}\text { Air, DI water }(1-10 \mathrm{~mm} \\
\text { above the sample })\end{array}$ \\
\hline
\end{tabular}

\subsection{Experimental Results}

\subsubsection{Scribing of copper thin film coated on a polyimide substrate}

3D non contact optical profilometer images of micro channels produced under air and water media are shown in figures 2 and 3. A reduction in the redeposition around the scribe edges in underwater laser scribing can be seen in figure 3. The variation in depth of the scribed microchannels on $\mathrm{Cu}$ films, against different laser energy maintained for ablation with three different wavelengths of 355 $\mathrm{nm}, 532 \mathrm{~nm}$ and $1064 \mathrm{~nm}$ and under air and DI water media is shown in figures 4-6. In all the cases, initially, with increase in the laser energy, the ablation depth increased but at higher levels of laser energy, either the increase in depth was saturated or the overall depth was decreased. For $95 \%$ overlap of the laser spot diameter and $45 \mathrm{~mJ}$ of energy, $532 \mathrm{~nm}$ wavelength laser resulted in a depth of $\sim 21 \mu \mathrm{m}$ compared to $\sim 12 \mu \mathrm{m}$ using $355 \mathrm{~nm}$ and $\sim 5 \mu \mathrm{m}$ using 1064 $\mathrm{nm}$. From figures 4 and 5 it could be seen that for energy less than $25 \mathrm{~mJ}$ both $355 \mathrm{~nm}$ and $532 \mathrm{~nm}$ resulted in same depth, as the laser energy increases depth reduced for 355 $\mathrm{nm}$ wavelength compared to $532 \mathrm{~nm}$. Under water scribing of $\mathrm{Cu}$ thin films show a reduction in the redeposition of the ablated material as seen in figure 3, but the channel depth achieved was less compared to air for 532 and $355 \mathrm{~nm}$ wavelengths as seen from figures 4 and 5 . However, for $1064 \mathrm{~nm} \mathrm{Cu}$ shows an opposite trend; the micro channel depth for underwater scribing was $\sim 7 \mu \mathrm{m}$ compared to $\sim 4$ $\mu \mathrm{m}$ produced in air. Increasing the height of DI water level above the workpiece has reduced the depth achieved. The change in width of the channel with respect to increase in laser energy was observed to be in few tens of microns.

\subsubsection{Scribing of aluminum thin film}

Figures 7-9 show the variation in depth of the scribed micro-channels on Al films, against different laser energies ablated with three different wavelengths of $355 \mathrm{~nm}, 532$ $\mathrm{nm}$ and $1064 \mathrm{~nm}$ in air and water mediums. It was observed that the ablation depth of micro channels produced in $\mathrm{Al}$ through underwater laser scribing was 6 times greater compared to the depth achieved in air for all the 3 wavelengths. It was observed that a depth of $\sim 100 \mu \mathrm{m}$ was achieved using both $355 \mathrm{~nm}$ and $532 \mathrm{~nm}$ with $4 \mathrm{~mJ}$ of energy and, 80 $\mu \mathrm{m}$ depth was achieved using $1064 \mathrm{~nm}$, while maintaining the same laser energy. The depth achieved for ablation in air medium, using $1064 \mathrm{~nm}$ wavelength with $4 \mathrm{~mJ}$ of energy was $10 \mu \mathrm{m}$ which is less compared to the $20 \mu \mathrm{m}$ depth achieved using $355 \mathrm{~nm}$ and $532 \mathrm{~nm}$ respectively. The ablation depth in air is seen to be increasing with increasing energy as seen in Figure 7-9, but laser ablation depths achieved in DI water medium tends to increase and start to reduce/ saturate after $\sim 5 \mathrm{~mJ}$ energy. Also the ablation depth was reducing as the DI water level above the workpiece increased for all the 3 wavelengths. The width of the micro channels obtained for energies up to $4 \mathrm{~mJ}$ was $\sim 160 \mu \mathrm{m}$ and was found to increase to $\sim 200 \mu \mathrm{m}$ for $7 \mathrm{~mJ}$ and remained unchanged with further increase of energy. This shows a slight increase in the depth of the channel with increase in energy but then after threshold energy the change in width was negligible for both air and DI water. 


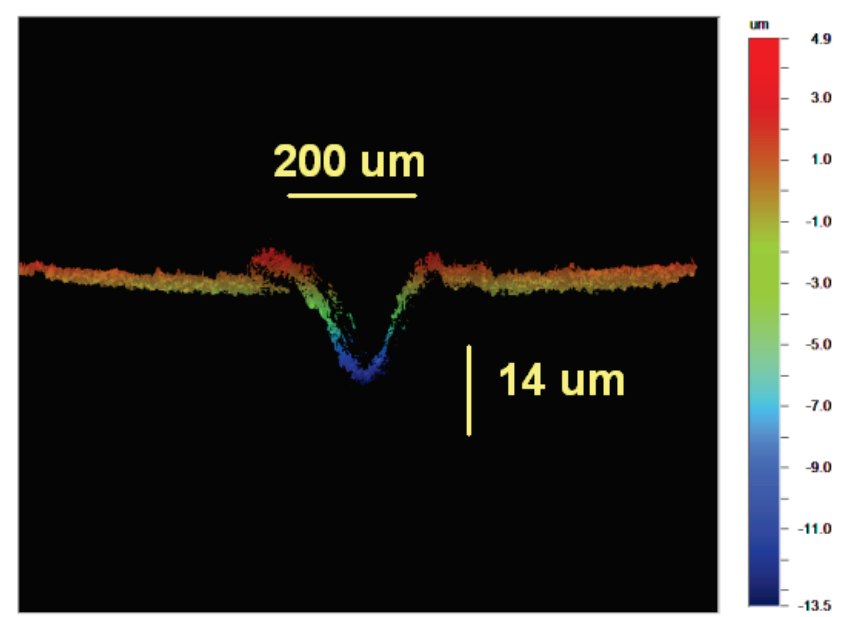

Fig. 2 3D image of the micro channel produced using $532 \mathrm{~nm}$ wavelength on $\mathrm{Cu}$ thin film coated on a polyimide substrate with $25 \mathrm{~mJ}$ energy in air

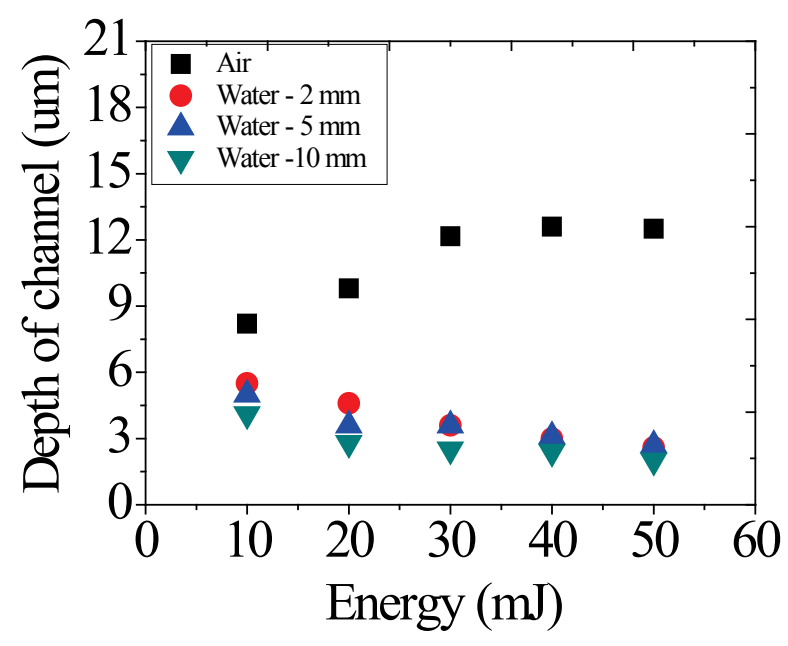

Fig. 4: Change in depth of copper thin film coated on polyimide substrate in air and different DI water levels for $355 \mathrm{~nm}$

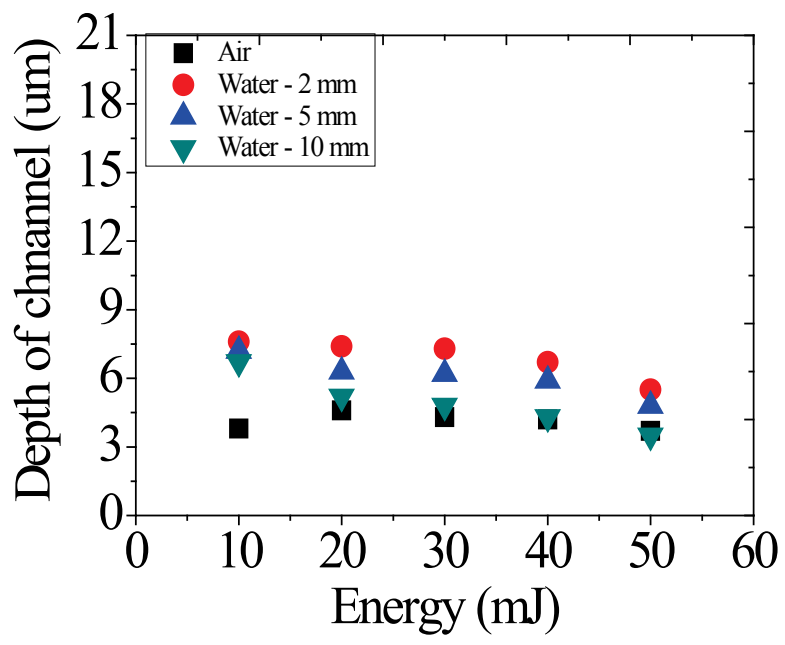

Fig. 6 Change in depth of copper thin film coated on polyimide substrate in air and different DI water levels for $1064 \mathrm{~nm}$

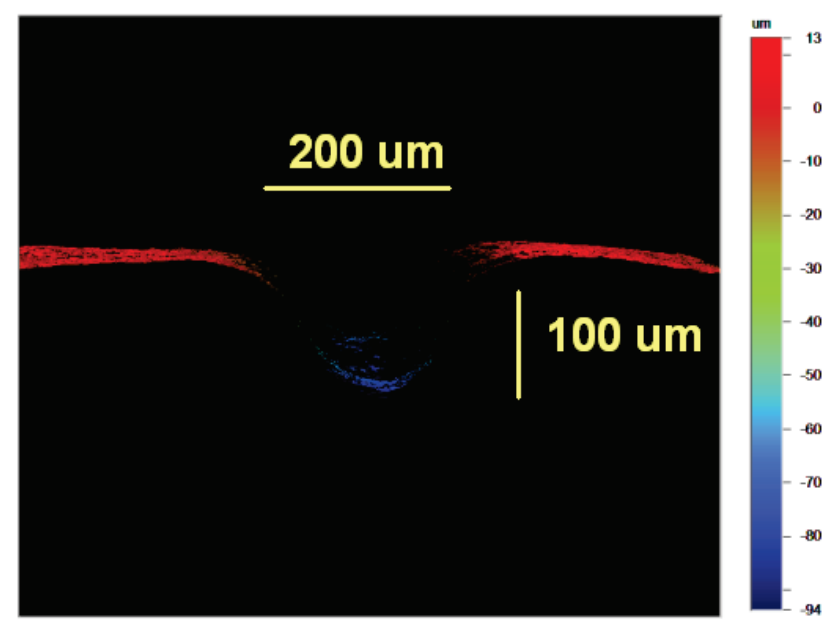

Fig. 3 3D image of the micro channel produced using $532 \mathrm{~nm}$ wavelength on $\mathrm{Al}$ thin film $5 \mathrm{~mJ}$ energy in DI water with $3 \mathrm{~mJ}$ energy

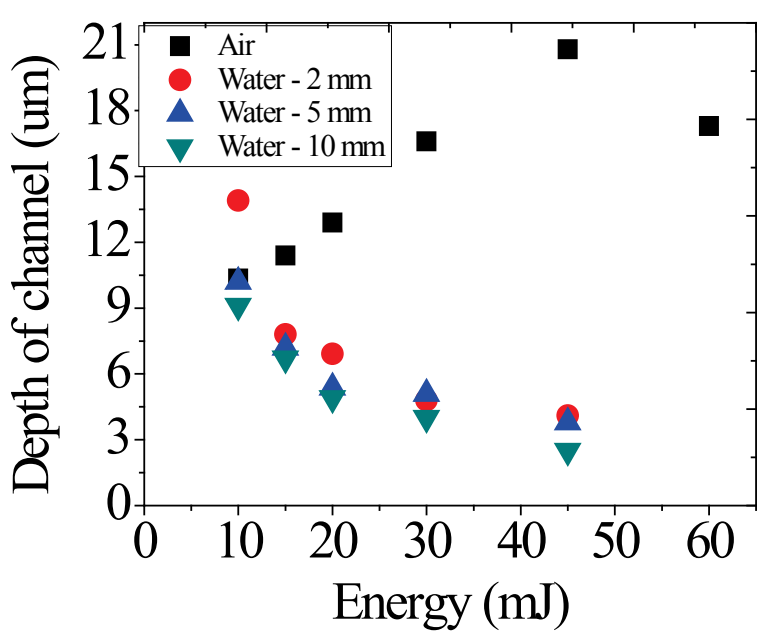

Fig. 5 Change in depth of copper thin film coated on polyimide substrate in air and different DI water levels for $532 \mathrm{~nm}$

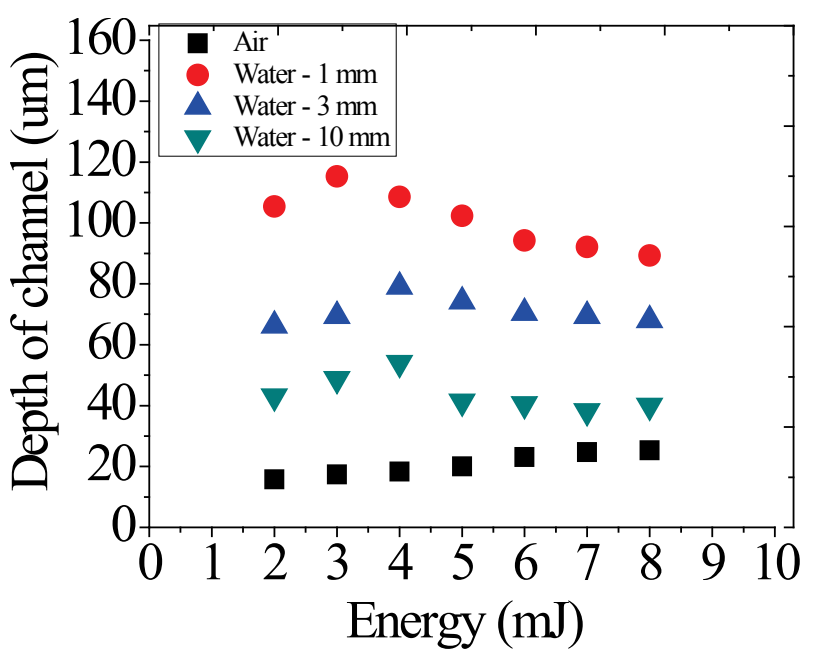

Fig. 7 Change in depth of aluminum thin film in air and different DI water levels for $355 \mathrm{~nm}$ 


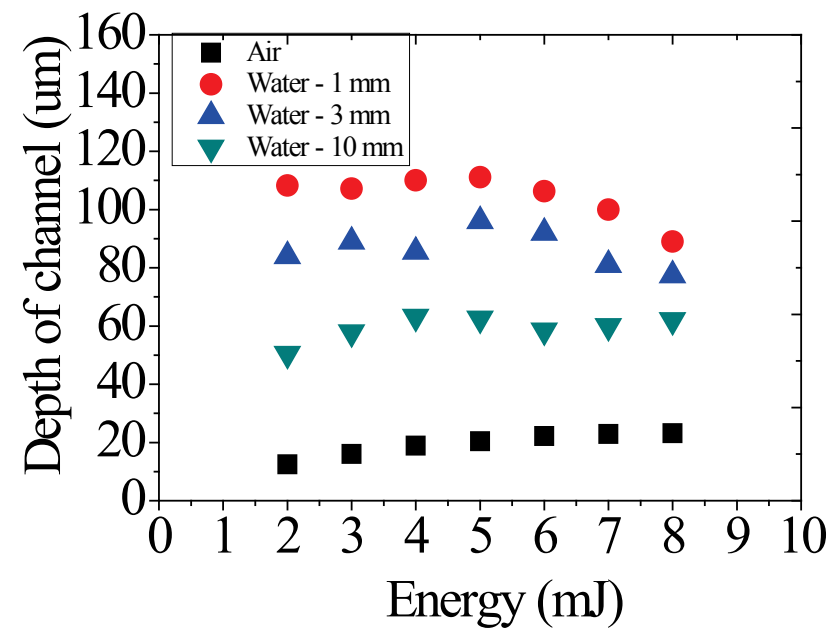

Fig. 8 Change in depth of aluminum thin film in air and different DI water levels for $532 \mathrm{~nm}$

\section{Theory of laser ablation of thin films}

In metals, light is absorbed by interaction with electrons. Excited electrons rise to conduction band and collide with lattice phonons, thereby transferring the absorbed energy to the lattice. Since the energy relaxation time is of the order of $10^{-13} \mathrm{~s}$ for metals [12], the optical energy can be considered to be converted instantly in to heat for ns-pulsed laser ablation. A thermal model of the laser impact on a solid $\mathrm{Cu} / \mathrm{Al}$ target is developed and studied to understand the phenomenological changes happening during the laser ablation process. Interaction of laser on the target surface increases the temperature at the contact interface, and the target will be able to melt, and even vaporize. The temperature distribution inside the target is calculated by means of the heat conduction equation (1). The absorption length in the target is in the order of $10 \mathrm{~nm}$.

$\frac{\partial T(x, t)}{\partial t}=\frac{\partial}{\partial x}\left[\left(\frac{k}{\rho C_{p}}\right) \frac{\partial T(x, t)}{\partial y}\right]+\frac{\alpha}{\rho C_{p}} I(x, t)$

where $\mathrm{T}$ represents the temperature inside the target, $x$ is the position from the surface; $\mathrm{t}$ is the time, $\mathrm{k}, \mathrm{C}_{\mathrm{p}}, \rho$ and $\alpha$ denotes the thermal conductivity, heat capacity, mass density and absorption coefficient of the target material, respectively. Laser irradiance $\mathrm{I}(x, t)$, a function of position and time as represented in equation (2), according to BeerLamberts law [12]

$$
I(x, t)=I_{o}(t) \exp (-\alpha x)[1-\check{\mathrm{R}}]
$$

where $\check{R}$ is reflectivity and $I_{o}(t)$ is the laser fluence at time t. When a pulse of laser beam is incident on the target surface, it interacts with the material and the lattice temperature increases accordingly. With the lattice temperature approaching the melting point of the target material, phase change is initiated and the temperature remains almost constant. After the solid to liquid phase transformation, the temperature again tends to increase and as it reaches the boiling point, the material starts to vaporize. The vapor pressure is given by Clausius-Clapeyron equation [12].

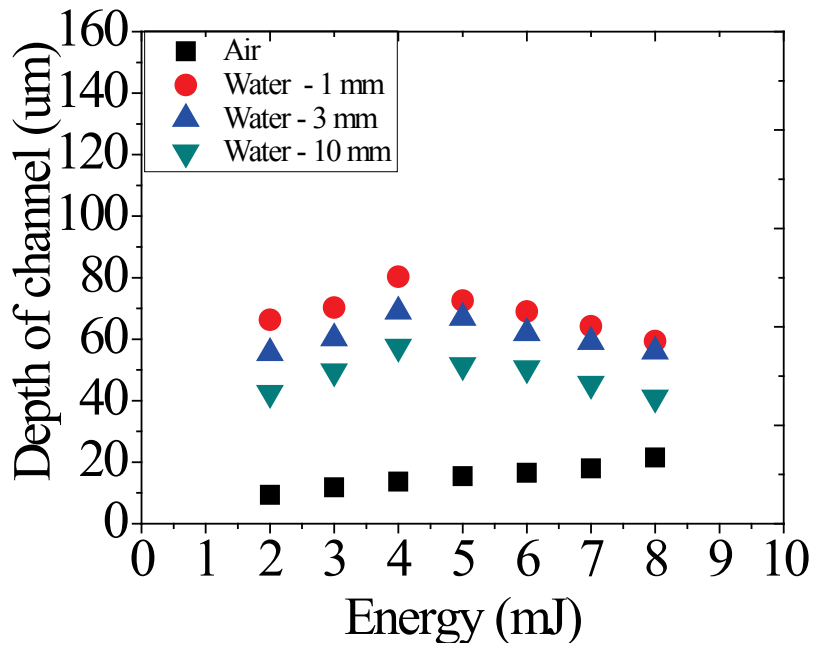

Fig. 9 Change in depth of aluminum thin film in air and different DI water levels for $1064 \mathrm{~nm}$

$p_{\text {vap }}\left(T_{s}\right)=p_{o} \exp \left[\frac{\Delta H_{l v}\left(T_{s}-T_{b}\right)}{R T_{s} T_{b}}\right]$

where $T_{s}$ and $T_{b}$ are the surface temperature and the normal boiling point respectively, pressure $\mathrm{p}_{0}$ is $100 \mathrm{KPa}, \Delta \mathrm{H}_{\mathrm{lv}}$ is the latent heat of vaporization, and $R$ is the gas constant. The flux of evaporated atoms is obtained from the vapor pressure at the surface temperature by the following equation [12].

$j_{\text {evap }}\left(T_{s}\right)=\frac{A_{p}\left(T_{s}\right)}{\sqrt{2 \pi M R T_{s}}}$

where $M$ is the molecular mass of the atoms $(\mathrm{kg})$ and $\rho$ the mass density $\left(\mathrm{kg} / \mathrm{m}^{3}\right)$. The surface recession rate due to evaporation is calculated from the flux of evaporated atoms using the equation 5 .

$R_{\text {recession }}=\frac{d x_{\text {evap }}}{d t}=\frac{j_{\text {evap }} M}{\rho}$

\subsection{Theoretical modeling Results for $\mathrm{Cu}$ thin film}

In order to understand the amount of heat conducted into the workpiece during the laser ablation process the temperature and recession rate have been calculated for 355, 532 and $1064 \mathrm{~nm}$ laser wavelength using a Gaussian shaped laser beam. Only a single laser pulse is considered for calculating the temperature and recession rate using the parameters tabulated in Table 2. Initially the copper target is considered to be at room temperature and the same is heated using the laser irradiance. In the current model, it is assumed that the absorption coefficient and the thermal conductivity of the target layer drop to zero as soon as the temperature of that particular layer reaches $9000 \mathrm{~K}$. This condition is applied in order to indicate that the material has been removed and the remaining heat energy will propagate to the next layer. The plasma electron temperature is calculated to be $9000 \mathrm{~K}$ which is assumed as the temperature at which ablation takes place.

The plasma electron temperature represented in equation (6) is estimated based on Boltzmann-Saha method [13] using the emission spectrum captured using LIBS technique shown in figure 10 [14]. 


$$
T_{e}=1.44 \frac{E_{2}-E_{1}}{\ln \left[\frac{I_{1} \lambda_{1} A_{2} g_{2}}{I_{2} \lambda_{2} A_{1} g_{1}}\right]}
$$

Where $T_{e}$ is the plasma electron temperature for copper sample $E_{1}$ and $E_{2}$ are excited energy levels $g_{1}$ and $g_{2}$ are statistical weights of excited energy levels $\mathrm{A} 1$ and $\mathrm{A}_{2}$ are the transition probabilities of states $\mathrm{I}_{1}$ and $\mathrm{I}_{2}$ are intensity of particular atomic species at $\lambda_{1}$ and $\lambda_{2}$ wavelengths respectively

Table 2 Parameters used in temperature estimation

\begin{tabular}{|c|c|c|c|c|c|}
\hline S No & $\lambda(\mathrm{nm})$ & $\mathrm{E}\left(\mathrm{cm}^{-1}\right)$ & $\mathrm{g}_{\mathrm{k}}$ & $\mathrm{A}_{\mathrm{k}}$ & $\mathrm{I}$ \\
\hline 1 & 510.5 & 30783.69 & 4 & $2 \times 10^{6}$ & 20933.7 \\
\hline 2 & 521.8 & 49942.05 & 6 & $7.5 \times 10^{7}$ & 56388.2 \\
\hline
\end{tabular}

Table 3 Parameters and specifications used in to modeling the influence of wavelength on $\mathrm{Cu}$ thin films

\begin{tabular}{ll}
\hline Parameters & Specifications \\
\hline Wavelength & $355 \mathrm{~nm}, 532 \mathrm{~nm}, 1064 \mathrm{~nm}$ \\
Pulse duration & $5 \mathrm{~ns}$ \\
Pulse Fluence & $20 \mathrm{~J} / \mathrm{cm}^{2}$ \\
Cu sample size & $60 \mu \mathrm{m} \times 10 \mu \mathrm{m}$ \\
Spot size (estimated) & $50 \mu \mathrm{m}$ \\
Reflectivity & $0.45(355), 0.65(532), 0.97(1064)$ \\
Absorption Coeffi- & $7.1 \times 10^{5} \mathrm{~cm}^{-1}(355), 5.8 \times 10^{5} \mathrm{~cm}^{-1}(532)$, \\
cient & $8.6 \times 10^{5} \mathrm{~cm}^{-1}(1064)$ \\
Copper vapourization & $2836 \mathrm{~K}$ \\
temperature & \\
\hline
\end{tabular}

\section{Discussion}

$\mathrm{Cu}$ and $\mathrm{Al}$ thin films were ablated with three different wavelengths $355 \mathrm{~nm}, 532 \mathrm{~nm}$ and $1064 \mathrm{~nm}$ respectively. To find the influence of wavelength and the ambient conditions on the characteristics of the scribed features, depth and width of micro channel are measured using 3D non contact profilometer. It was observed that for both the metals with increase in energy, ablation depth increases but at higher energy, depth of channel remains unchanged. In a study conducted [15], it was found that the shorter wavelength (in the UV region) caused a large absorption of laser energy by the surface and led to a higher temperature rise at the interface leading to a lower threshold fluence value. This is supported by the fact that the surface reflectivity for $\mathrm{Cu}$ is 0.45 for $355 \mathrm{~nm}, 0.62$ for $532 \mathrm{~nm}$ and 0.97 for 1064 $\mathrm{nm}$, and the absorption coefficient is $7.1 \times 10^{5} \mathrm{~cm}^{-1}$ for 355 $\mathrm{nm}, 6.1 \times 10^{5} \mathrm{~cm}^{-1}$ for $532 \mathrm{~nm}$, and $8.1 \times 10^{5} \mathrm{~cm}^{-1}$ for 1064 $\mathrm{nm}$ [16]. Since the absorption depth is high for $532 \mathrm{~nm}$, it is possible to achieve a greater depth in comparison with 355 $\mathrm{nm}$ and $1064 \mathrm{~nm}$ for same energy. Moreover, at higher energies the micro channels produced using $355 \mathrm{~nm}$ wavelength showed a higher heat affected zone around the channel edges, which is an indication of plasma formation and subsequent heating of the workpiece, which is the cause of reduction in the depth. The formation of plasma above the processing surface can lead to shielding of the work piece surface from the incident beam, thereby preventing the incident beam from coupling to the work piece surface. Previous experimental investigations into the

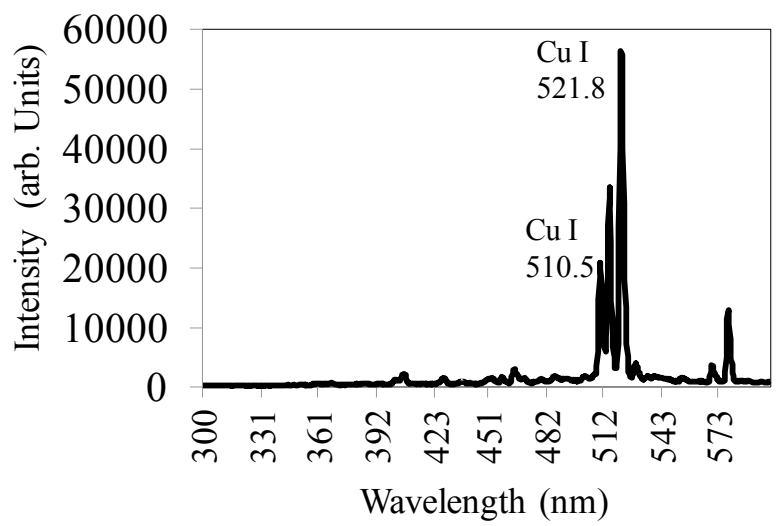

Fig. 10 Optical emission spectra obtained using LIBS technique for copper film coated with polyimide substrate using $15 \mathrm{~mJ}$ energy

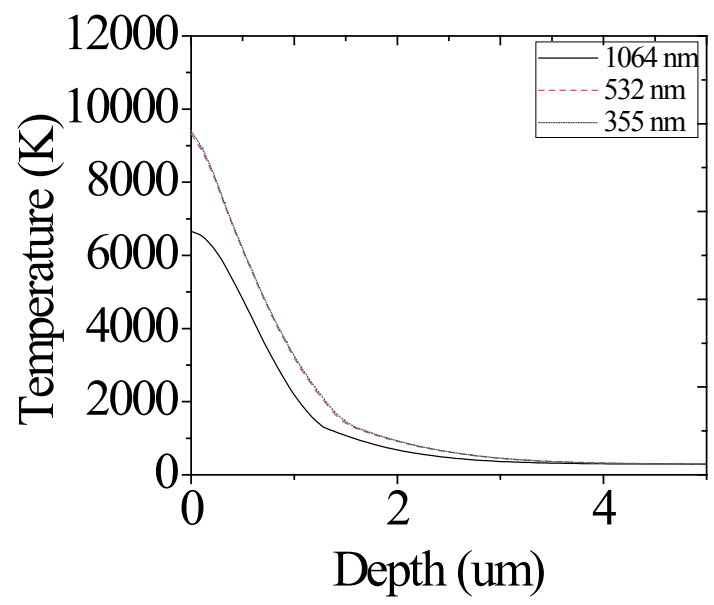

Fig. 11(a) Temperature plot for $\mathrm{Cu}$ along the depth profile for 3 different wavelengths at $20 \mathrm{~J} / \mathrm{cm}^{2}$ fluece

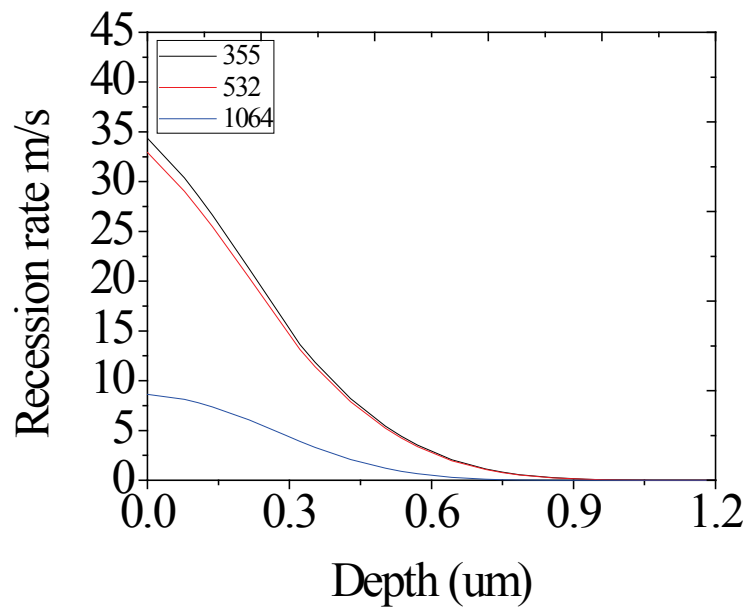

Fig. 11(b) Recession rate for $\mathrm{Cu}$ along the depth profile for 3 different wavelengths: $1064 \mathrm{~nm}, 532 \mathrm{~nm}$ and $355 \mathrm{~nm}$

interaction of a short wavelength laser with various metals and ceramics have shown that the formation of plasma over the work piece surface can lead to decoupling of the incident beam from the work piece surface [8]. In underwater scribing, as the DI water level above the work piece decreases, ablation rate increases resulting in increased depth of the scribed channel. 


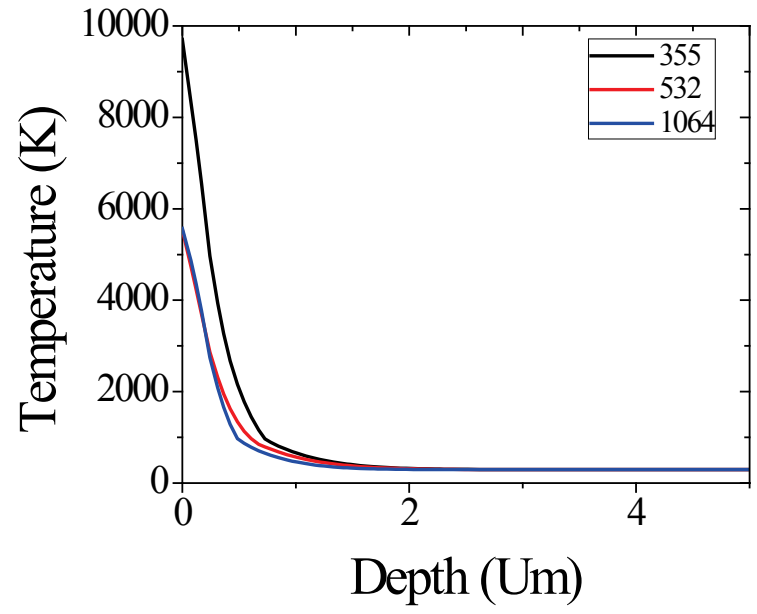

Fig 12: Temperature plot for $\mathrm{Al}$ along the depth profile for 3 different wavelengths at $5 \mathrm{~J} / \mathrm{cm}^{2}$ fluence

For a DI water level $\mathrm{h}=1 \mathrm{~mm}$, depth of micro channel is maximum. Increasing the DI water height leads to absorption of laser energy by the medium especially for $1064 \mathrm{~nm}$, causing a reduction in the available energy at the target. No re-deposition at the edges is observed when micro scribing is done in DI water medium. If the work piece is covered with a DI water layer, the plasma is confined and its expansion is delayed. Therefore, the induced pressure is 4 to 10 times greater than the corresponding one obtained in air medium and the shock wave duration is $2-3$ times longer than in the air regime at the same power density [17]. This results in higher material removal rates resulting in high ablation depth. Copper has less reflectivity at shorter wavelengths; reflectivity also plays a role in underwater laser scribing. The sudden increase in temperature due to the laser pulse induces shock waves caused by the cavitation bubbles that expand and collapse forming a high pressure liquid jet. These shock waves are reflected by the work piece and are reflected back from the air-DI water interface and increase the impact on the work piece. Because of high reflectivity of Al, depth in DI water is higher for all wavelengths, whereas $\mathrm{Cu}$ has lesser reflectivity at shorter wavelength which explains the reduced depth. The micro channel depth is increasing for $\mathrm{Al}$ with energy but after certain value of laser energy $(5 \mathrm{~mJ}-6 \mathrm{~mJ})$ depth decreases. It is because of the parasitic plasma occurring in confining DI water. It has been reported that above a 10 $\mathrm{GW} / \mathrm{cm}^{2}$ laser intensity threshold, a saturation of the peak pressure is shown to occur while the shock wave duration is reduced by parasitic plasma occurring in the confining DI water [17]. This phenomenon occurs at the surface of the DI water rather than within the water volume which leads to absorption of the incident laser energy, and the laser fluence reaching the target gradually decreases.

It was observed experimentally using $20 \mathrm{~J} / \mathrm{cm}^{2}$ fluence for 3, 4 and 5 pulses, the depth achieved is $3.6 \mu \mathrm{m}, 4.3 \mu \mathrm{m}$ and $5.2 \mu \mathrm{m}$, respectively. From this data, it is inferred that for one pulse the depth is $\sim 1 \mu \mathrm{m}$. The depth achieved for $532 \mathrm{~nm}$ from the present model at $3000 \mathrm{~K}$ is thus validated with the experimental data. Figure 11(a) shows the temperature profile estimated using equation 1 for the 3 wavelengths for $\mathrm{Cu}$ sample. In the developed model, as we have not incorporated the effect of plasma and the change in material properties with temperature, both $355 \mathrm{~nm}$ and 532 $\mathrm{nm}$ laser wavelengths follow the same trend, but since the reflectivity of $1064 \mathrm{~nm}$ for same fluence is high the temperature obtained at the surface is less than $355 \mathrm{~nm}$ and 532 $\mathrm{nm}$. Figure 11(b) shows the variation of the recession rate with the depth for copper sample at different wavelengths. From the figure it could be observed that the trend of variation of recession rate is similar to that of the temperature. This observation is also proved experimentally with the depth results obtained for $1064 \mathrm{~nm}$ which is lesser compared to shorter wavelengths. Figure 12 shows the temperature profile for Al sample for 3 different wavelengths. The depth attained at boiling point temperature is less compared to $\mathrm{Cu}$.

This developed model can be further improved by incorporating the effect of temperature on the material properties of the work piece and the plasma absorption phenomenon in order to predict the laser material interaction with more accuracy.

\section{Conclusions}

Influence of varying laser wavelengths namely $355 \mathrm{~nm}$, $532 \mathrm{~nm}$ and $1064 \mathrm{~nm}$ on the characteristics of the micro channels generated during underwater scribing of metal thin films was studied. Greater depth for Al work piece was obtained in ablation in underwater medium than in air medium. As DI water level decreases, ablation rate becomes higher and the depth of channel increases. At DI water level, $\mathrm{h}=1 \mathrm{~mm}$ depth of micro channel was maximum. With increase in DI water level the depth of the scribe channel reduces and this is attributed to the reduction in the plasma temperature due to quenching by excess water and thus reduction in the plasma pressure. With increase in energy of pulsed laser beam, the medium starts to break down due to parasitic breakdown at the interface of water and air and depth of micro channel remains unchanged at higher energies

Reflectivity of the work piece also results in the increased depth of the micro channels in underwater scribing due to multiple reflections of the shockwaves between the workpiece surface and the air-water interface. It was observed that shorter wavelength ablates more effectively compared to longer wavelength in DI water medium in case of $\mathrm{Al}$, whereas for $\mathrm{Cu}$, longer wavelength ablates more material in DI water medium compared to air. As energy increases, the width of channel also increases initially, but after $6 \mathrm{~mJ}$ of energy the change in width was limited to few microns. Melt pool was observed in dry (air) ablation and not in underwater ablation.

\section{Acknowledgments}

This work is partly supported by the grant-in aid from the Indian Space Research Organization (ISRO) (EDD/1415/151/ISRO/NILE).

The authors are grateful to the members of micro electronics and MEMS lab, Department of electrical engineering and physical metallurgy lab, Department of Metallurgical and Materials Engineering, IIT Madras for providing facilities for characterization. 


\section{References}

[1] M. J Jackson and W. O'Neill: Journal of Materials Processing Technology, 142, (2003) 517.

[2] J.C Aurich, D. Dornfeld, P. J Arrazola, V. Franke, L. Leitz, and S. Min: CIRP Annals-Manufacturing Technology, 58, (2009) 519.

[3] R. Fabbro, J. Fournier and P. Ballard, D. Devaux, J. Virmont: Journals of Applied Physics, 68, (1990) 775.

[4] W. T. Chien, and S. C Hou.: The International Journal of Advanced Manufacturing Technology, 33, (2007) 308.

[5] K. Hock, A. Benedikt, and R Hellmann: Physics Procedia, 39, (2012) p. 225.

[6] N. Krstulović, S. Shannon, R. Stefanuik and C. Fanara: The International Journal of Advanced Manufacturing Technology, 69, (2013) 1765.

[7] J Lu, R. Q. Xu, X. Chen, Z. H. Shen, X. W. Ni, S. Y. Zhang, and C. M. Gao: Journal Of Applied Physics, 95, (2004) 3890.

[8] W. H. Kang, H. Lee, and A. J. Welch: Journal of Applied Physics, 103, (2008) 083101.

[9] L. Tunna, A. Kearns, W. O'neill, and C. J. Sutcliffe: Optics \& Laser Technology, 33, (2001) 135-143.

[10] S. Nammi, N. J. Vasa, G Balaganesan, S Gupta and A. C. Mathur: Journal of Micro/Nanolithography, MEMS MOEMS, 14, (2015) 044503.

[11] J. J Chang, and B. E. Warner: Applied physics letters, 69, (1996) 473.

[12] A. Bogaerts, C Zhaoyang, R Gijbels, and A.Vertes: Atomic Spectroscopy, 58, (2003) 1867.

[13] A. Descoeudres, C. Hollenstein, R. Demellayer, and G. Wälder: Journal Of Materials Processing Technology, 149, (2004) 184.

[14] N. Aparna, N. J. Vasa, R. Sarathi, and J. Sundara Rajan: Applied Physics A, 117, (2014) 281.

[15] J. M. Lee, C. Curran, K. G. Watkins: Applied Physics A, 73, (2001) 219.

[16] D.W. Lynch, W.R. Hunter: "Handbook of Optical Constants of Solids" ed. by E.D. Palik, (Academic Press, 1985) p. 280.

[17] L. Berthe, R. Fabbro, P. Peyre, L. Tollier, and E. Bartnicki: Journals of Applied Physics, 82, (1997) 2826.

(Received: May 26, 2015, Accepted: January 17, 2016) 